\title{
General note about the special issue
}

Data presented on government and institutional websites may differ from the data appearing in this issue due to the dynamic nature of this crisis and the data reported, as well as differences in measurement definitions used. 